\title{
Behaviors and Numerical Simulations of Malaria Dynamic Models with Transgenic Mosquitoes
}

\author{
Xiongwei Liu, ${ }^{1}$ Junjun $\mathrm{Xu}^{2}{ }^{2}$ Xiao Wang, ${ }^{1}$ and Lizhi Cheng ${ }^{1}$ \\ ${ }^{1}$ College of Science, National University of Defense Technology, Changsha, Hunan 410073, China \\ ${ }^{2}$ Xichang Satellite Launch Center, Xichang, Sichuan 615000, China \\ Correspondence should be addressed to Xiongwei Liu; csxw2010@163.com
}

Received 21 November 2013; Accepted 4 January 2014; Published 20 February 2014

Academic Editor: Bingwen Liu

Copyright (c) 2014 Xiongwei Liu et al. This is an open access article distributed under the Creative Commons Attribution License, which permits unrestricted use, distribution, and reproduction in any medium, provided the original work is properly cited.

\begin{abstract}
The release of transgenic mosquitoes to interact with wild ones is a promising method for controlling malaria. How to effectively release transgenic mosquitoes to prevent malaria is always a concern for researchers. This paper investigates two methods of releasing transgenic mosquitoes and proposes two epidemic models involving malaria patients, anopheles, wild mosquitoes, and transgenic mosquitoes based on system of continuous differential equations. A basic reproduction number $\mathbf{R}_{0}$ is defined for the models and it serves as a threshold parameter that predicts whether malaria will spread. By theoretical analysis of the dynamic behaviors of the models and numerical simulations, it is verified that malaria can be effectively controlled by the opportune release of transgenic mosquitoes; that is, when $\mathbf{R}_{0} \leq 1$, malaria will disappear; when $\mathbf{R}_{0}>1$, malaria will become an endemic disease in the target field.
\end{abstract}

\section{Introduction}

Malaria is an infectious mosquito-borne disease. No vaccine and no specific drugs are available for malaria because plasmodium which causes malaria have become increasingly resistant to drugs. An effective way to prevent malaria is to control mosquitoes. Therefore, scientists hope to use genetic engineering technology to release transgenic mosquitoes which cannot transmit malaria to cut off malaria transmission chains [1-4].

But function laws of transgenic mosquitoes released to prevent malaria transmission can only be acquired from a large number of experimental data in consideration of the influence on other species, ecological environment, possible risks, and involvement [4-6]. Therefore, it has a practical significance to establish dynamic models which can reflect the change laws of many factors and study the dynamic behaviors of the models to recognize the role of transgenic mosquitoes in malaria transmission in terms of all statuses of releasing transgenic mosquitoes.

Many researchers have made a lot of theoretical researches on the role of transgenic mosquitoes in decreasing anopheles and preventing malaria transmission. Some wonderful mathematical models are presented. For example, the possibilities of replacing wild mosquitoes with transgenic ones released in different ways were considered, malaria transmission model was established, and the existence and stability of the disease-free equilibrium points were discussed with the aid of Floquet theory in [7]. The epidemic dynamic models of malaria for sawtooth animals were established and behaviors of each infection stage were discussed with numerical simulations in [8]. The detailed analysis and discussion on how to prevent malaria by mathematical models were presented in [9]. Li has long been engaged in the research in this field. He mainly investigated the impact of the environment, wild mosquitoes, genetically modified mosquitoes, and so forth and formulated the stage-structured discrete-time and continuous-time mathematical models for interacting wild and transgenic mosquito populations [10-14].

However, at present, many existing models are generally limited to discuss the role of population characters of two classes of mosquitoes, technology development, and the environmental factors that prevent malaria and do not 
consider other more factors, such as infected patients. For the analysis of the infected population controlled by transgenic mosquitoes in malaria transmission, these works are not enough. In order to better investigate the actual role of transgenic mosquitoes in malaria transmission and make the models more realistic, according to the current international research results which have been obtained, we establish a population dynamic model and an epidemic dynamic model involving patients, anopheles, wild mosquitoes, and transgenic mosquitoes released in two different ways based on systems of differential equations. With the aid of qualitative theory, we study behaviors of these models with numerical simulations and provide the conditions under which equilibriums of these models are asymptotically stable. Based on the researches on modeling dynamical behaviors, the impacts of various parameters changes in the models on malaria transmission and the two methods of releasing transgenic mosquitoes on controlling the amount of malaria patients in practice are investigated with numerical simulations.

This paper is organized as follows. In Section 2, we firstly assume that transgenic mosquitoes are released at a fixed proportion and interact with wild mosquitoes. Secondly, we assume that transgenic mosquitoes are released at a changeable proportion. Then the existence of all possible equilibriums is investigated and their stabilities are studied. In Section 3, numerical simulations are supplemented to demonstrate the results in Section 2. In Section 4, the brief discussions of our findings and prospects are presented.

\section{The Models and Stability Analyses}

In 1927, Kermack and Mckendrick established an epidemic model, namely, KM model [15]. In this paper, based on KM assumptions and the epidemic model in [16, 17], we will formulate and discuss two dynamic models with male transgenic mosquitoes which do not suck blood [18] released in two different ways.

\subsection{The Dynamic Model with Transgenic Mosquitoes Released} at a Fixed Proportion. For simplicity, we first consider the model with transgenic mosquitoes released into the field of mosquitoes at a fixed proportion. We assume that the population size in the target field is large and KM assumptions of epidemic model are met. The proportion at which transgenic mosquitoes are released is a constant $a(0 \leq a<1)$. Then, we can establish a system of differential equations:

$$
\begin{gathered}
\frac{d x}{d t}=\beta y(1-x)-\gamma x, \\
\frac{d y}{d t}=\alpha x(1-a-y)-\mu y-c a y,
\end{gathered}
$$

where $\beta, \gamma, \alpha, \mu, c$ are positive constants. The descriptions of these parameters in system (1) are shown in Table 1.

\begin{tabular}{|c|c|}
\hline Parameter & Description \\
\hline$a$ & $\begin{array}{l}\text { Proportion at which transgenic mosquitoes are } \\
\text { released }\end{array}$ \\
\hline$\beta$ & Incidence rate of malaria due to biting \\
\hline$\alpha$ & $\begin{array}{l}\text { Efficiency of infection in mosquitoes by biting } \\
\text { patient }\end{array}$ \\
\hline$\mu$ & Death rate of anopheles \\
\hline$\gamma$ & Recovery rate of patients \\
\hline$c$ & $\begin{array}{l}\text { Decrement rate of anopheles due to transgenic } \\
\text { mosquitoes bred by transgenic mosquitoes and } \\
\text { anopheles }\end{array}$ \\
\hline$x(t)$ & Proportion of patients at $t$ time \\
\hline$y(t)$ & Proportion of anopheles at $t$ time \\
\hline
\end{tabular}

TABLE 1: Descriptions of the parameters in system (1).

Let $\mathbf{R}_{\mathbf{0}}^{\mathbf{1}}=\alpha \beta(1-a) / \gamma(\mu+c a)$. System (1) has a unique equilibrium $(0,0)$ for $\mathbf{R}_{\mathbf{0}}^{\mathbf{1}} \leq 1$ and the endemic equilibrium $\left(x_{0}, y_{0}\right)$ exists if and only if $\mathbf{R}_{\mathbf{0}}^{\mathbf{1}}>1$; here

$$
x_{0}=\frac{\gamma(c a+\mu)\left(\mathbf{R}_{\mathbf{0}}^{\mathbf{1}}-1\right)}{\alpha \beta(1-a)+\alpha \gamma}, \quad y_{0}=\frac{\gamma(c a+\mu)\left(\mathbf{R}_{\mathbf{0}}^{\mathbf{1}}-1\right)}{\beta(a c+\alpha+\mu)} .
$$

For system (1), we can obtain the following conclusion.

Theorem 1. (i) The equilibrium $(0,0)$ is locally asymptotically stable if $\mathbf{R}_{\mathbf{0}}^{\mathbf{1}}<1$. (ii) The endemic equilibrium $\left(x_{0}, y_{0}\right)$ is locally asymptotically stable and $(0,0)$ is unstable if $\mathbf{R}_{\mathbf{0}}^{\mathbf{1}}>1$; here $\left(x_{0}, y_{0}\right)$ is the same as that in $(2)$.

Proof. (i) The linearization form of system (1) about $(0,0)$ is

$$
\begin{gathered}
\frac{d x}{d t}=-\gamma x+\beta y, \\
\frac{d y}{d t}=\alpha(1-a) x-(\mu+c a) y .
\end{gathered}
$$

The corresponding characteristic equation of (3) is

$$
\lambda^{2}+(\gamma+\mu+c a) \lambda+\gamma(a c+\mu)\left(1-\mathbf{R}_{\mathbf{0}}^{\mathbf{1}}\right)=0 .
$$

Solving (4), we can get two characteristic roots:

$\lambda_{1,2}=\frac{-(a c+\gamma+\mu) \pm \sqrt{(a c+\gamma+\mu)^{2}-4 \gamma(a c+\mu)\left(1-\mathbf{R}_{0}^{1}\right)}}{2}$.

From (5), it is easy to see when $\mathbf{R}_{0}^{\mathbf{1}}<1$, the real parts of (5) are all negative. Therefore, the equilibrium $(0,0)$ is locally asymptotically stable. It implies that malaria will eventually disappear. When $\mathbf{R}_{\mathbf{0}}^{\mathbf{1}}>1,(0,0)$ is saddle point; it is unstable.

(ii) Linearizing system (1) about $\left(x_{0}, y_{0}\right)$ yields

$$
\begin{gathered}
\frac{d x}{d t}=\left(-\beta y_{0}-\gamma\right)\left(x-x_{0}\right)+\beta\left(1-x_{0}\right)\left(y-y_{0}\right), \\
\frac{d y}{d t}=\alpha\left(1-a-y_{0}\right)\left(x-x_{0}\right)-\left(\alpha x_{0}+\mu+c a\right)\left(y-y_{0}\right) .
\end{gathered}
$$


The corresponding characteristic equation of (6) is

$$
\begin{array}{r}
\left(\lambda+\beta y_{0}+\gamma\right)\left(\lambda+\alpha x_{0}+\mu+c a\right) \\
-\alpha \beta\left(1-x_{0}\right)\left(1-a-y_{0}\right)=0 .
\end{array}
$$

Solving (7), we can get two characteristic roots

$$
\lambda_{1,2}=\frac{-\left(\beta y_{0}+\gamma+\alpha x_{0}+\mu+c a\right) \pm \sqrt{\Delta}}{2},
$$

where

$$
\begin{gathered}
\Delta=\left(\beta y_{0}+\gamma+\alpha x_{0}+\mu+c a\right)^{2}-4 K \\
K=\left(\beta y_{0}+\gamma\right)\left(\alpha x_{0}+\mu+c a\right)-\alpha \beta\left(1-x_{0}\right)\left(1-a-y_{0}\right) .
\end{gathered}
$$

From (2) and (9), it follows that

$$
K=(a c \gamma+\gamma \mu)\left(\mathbf{R}_{\mathbf{0}}^{1}-1\right) .
$$

Therefore, if $\mathbf{R}_{\mathbf{0}}^{\mathbf{1}}>\mathbf{1}$, the real parts of (8) are all negative, which indicates that the endemic equilibrium $\left(x_{0}, y_{0}\right)$ is locally asymptotically stable. It implies that malaria will be popular in the target field, but it will not be massively diffusive. Hence, Theorem 1 is completed.

Remark 2. When $a=0$, system (1) becomes the model in [16]. From above results, when $\mathbf{R}_{\mathbf{0}}^{\mathbf{1}}<1$, we can see that malaria are prevented effectively by releasing transgenic mosquitoes. From $\mathbf{R}_{\mathbf{0}}^{\mathbf{1}}=\alpha \beta(1-a) / \gamma(\mu+c a)$, as long as we increase the value of $a$ or $c$, that is, increase transgenic mosquitoes or decrease anopheles, malaria can be prevented in the target field.

\subsection{The Dynamic Model with Transgenic Mosquitoes Released} at a Changeable Proportion. Releasing transgenic mosquitoes at a fixed proportion is more difficult than being carried out in reality and also does not accord with the actual situation. Therefore, in this section we introduce a changeable proportion $z(t)$, which is the proportion of transgenic mosquitoes to mosquito population at time $t$. At time $t$, the proportion of anopheles is $y(t)$ and the proportion of susceptible mosquitoes is $1-y(t)-z(t) . x(t)$ is the proportion of infected persons; then $1-x(t)$ is the proportion of susceptible persons. Similarly, we can establish the following model:

$$
\begin{gathered}
\frac{d x}{d t}=\beta y(1-x)-\gamma x, \\
\frac{d y}{d t}=\alpha x(1-y-z)-\mu y-c z y, \\
\frac{d z}{d t}=\delta_{1} z y+\delta_{2} z(1-y-z)-\omega z,
\end{gathered}
$$

where $\beta, \gamma, \alpha, \mu, c, \delta_{1}, \delta_{2}, \omega$ are constants. The description of these parameters in system (11) is shown in Table 2.

Assume that $\delta_{1}=\delta_{2}=\delta$, system (11) has four fixed points which are malaria-free equilibriums $E_{0}(0,0,0), E_{1}\left(0,0, z_{1}\right)$, and endemic equilibriums $E_{2}\left(x_{2}, y_{2}, z_{2}\right)$ and $E_{3}\left(x_{3}, y_{3}, 0\right)$, respectively; here

$$
\begin{gathered}
x_{2}=\frac{\gamma c \omega+\alpha \beta \omega-\gamma \mu \delta-\gamma c \delta}{\alpha \beta \omega+\alpha \delta \gamma}, \\
y_{2}=\frac{\gamma c \omega+\alpha \beta \omega-\gamma \mu \delta-\gamma c \delta}{\alpha \beta \omega+\mu \delta \beta+c \delta \beta-c \beta \omega}, \\
z_{1}=z_{2}=1-\frac{\omega}{\delta}, \quad x_{3}=\frac{\alpha \beta-\gamma \mu}{\alpha(\beta+\gamma)}, \quad y_{3}=\frac{\alpha \beta-\gamma \mu}{\beta(\alpha+\mu)} .
\end{gathered}
$$

Remark 3. When $\delta_{1} \neq \delta_{2}$, we can still obtain (13), but $z_{1}=$ $z_{2}=1-\omega / \delta_{2}$ and the expressions of (12) are very complicated.

For system (11), since the equilibrium $E_{0}(0,0,0)$ and endemic equilibrium $E_{3}\left(x_{3}, y_{3}, 0\right)$ are not meaningful for reality, we just consider the stability of the equilibrium $E_{1}\left(0,0, z_{1}\right)$ and $E_{2}\left(x_{2}, y_{2}, z_{2}\right)$. Taking $\mathbf{R}_{0}^{2}=\alpha \beta \omega / \gamma(c \delta+\delta \mu-$ $\omega)$, for system (11), we can obtain the following conclusion.

Theorem 4. Assuming that $\delta_{1}=\delta_{2}=\delta$ and $\omega<\delta$ which implies the birth rate of the transgenic mosquitoes is larger than the death rate, one has

(i) the equilibrium $E_{1}\left(0,0, z_{1}\right)$ is locally asymptotically stable if $\mathbf{R}_{\mathbf{0}}^{\mathbf{2}}<1$,

(ii) the endemic equilibrium $E_{2}\left(x_{2}, y_{2}, z_{2}\right)$ is locally asymptotically stable if $\mathbf{R}_{\mathbf{0}}^{2}>1$; here $z_{1}, x_{2}, y_{2}, z_{2}$ are the same as those in (12) and (13).

Proof. The matrix corresponding to the linearization form of system (11) on $\left(0,0, z_{1}\right)$ is

$$
A=\left(\begin{array}{ccc}
-\gamma & \beta & 0 \\
\alpha\left(1-z_{1}\right) & -\mu-c z_{1} & 0 \\
0 & \delta z_{1}-\delta z_{1} & \delta-2 \delta z_{1}-\omega
\end{array}\right)
$$

Its characteristic equation is

$$
|\lambda E-A|=\left|\begin{array}{ccc}
\lambda+\gamma & -\beta & 0 \\
\alpha\left(z_{1}-1\right) & \lambda+\mu+c z_{1} & 0 \\
0 & \delta z_{1}-\delta z_{1} & \lambda-\delta+2 \delta z_{1}+\omega
\end{array}\right|=0 .
$$

Solving the above equation, we can get its characteristic roots

$$
\begin{gathered}
\lambda_{1}=-\omega+\left(1-2 z_{1}\right) \delta_{2}, \\
\lambda_{2,3}=\left(-\gamma-\mu-c z_{1}\right. \\
\left.\quad \pm \sqrt{\left(\gamma+\mu+c z_{1}\right)^{2}-4\left(-\alpha \beta+\gamma \mu+(\alpha \beta+c \gamma) z_{1}\right)}\right) \\
\quad \times(2)^{-1} .
\end{gathered}
$$

From (13) and $\omega<\delta$, we have

$$
\lambda_{1}=-\omega+\left(1-2 z_{1}\right) \delta=\omega-\delta<0 .
$$


TABLE 2: Description of the parameters in system (11).

\begin{tabular}{ll}
\hline Parameter & Description \\
\hline$\beta$ & Incidence rate of malaria due to biting \\
$\alpha$ & Efficiency of infection in mosquitoes by biting patients \\
$\mu$ & Death rate of anopheles \\
$\gamma$ & Recovery rate of patients \\
$c$ & Decrement rate of anopheles due to transgenic mosquitoes bred by transgenic mosquitoes and anopheles \\
$\omega$ & Death rate of transgenic mosquitoes \\
$\delta_{1}$ & Birth rate of transgenic mosquitoes bred by transgenic mosquitoes and wild anopheles \\
$\delta_{2}$ & Birth rate of transgenic mosquitoes bred by transgenic mosquitoes and wild susceptible mosquitoes \\
$x(t)$ & Proportion of patients at $t$ time \\
$y(t)$ & Proportion of anopheles at $t$ time \\
$z(t)$ & Proportion of transgenic mosquitoes released at time $t$ \\
\hline
\end{tabular}

If $\operatorname{Re} \lambda_{2,3}<0$, system (11) is locally asymptotically stable on $\left(0,0, z_{1}\right)$. In fact, from (13) and $\lambda_{2,3}$ in (16), we have

$$
-\alpha \beta+\gamma \mu+(\alpha \beta+c \gamma) z_{1}=\alpha \beta \frac{\omega}{\delta}\left(1-\mathbf{R}_{0}^{2}\right)>0 .
$$

That is to say, $\operatorname{Re} \lambda_{2,3}<0$. Therefore, system (11) is locally asymptotically stable on $\left(0,0, z_{1}\right)$. It implies that malaria and anopheles will eventually disappear.

Now we consider behaviors of the local asymptotical stability of system (11) on $\left(x_{2}, y_{2}, z_{2}\right)$ which is a positive root under the condition $\mathbf{R}_{\mathbf{0}}^{2}>1$.

The matrix corresponding to the linearization form of system (11) on $\left(x_{2}, y_{2}, z_{2}\right)$ is

$$
A=\left(\begin{array}{ccc}
-\beta y_{2}-\gamma & \beta\left(1-x_{2}\right) & 0 \\
\alpha\left(1-y_{2}-z_{2}\right) & -\alpha x_{2}-\mu-c z_{2} & -\alpha x_{2}-c y_{2} \\
0 & 0 & \delta-\omega-2 \delta z_{2}
\end{array}\right) .
$$

The characteristic equation of this matrix is

$$
\begin{aligned}
& |\lambda E-A| \\
& \quad=\left|\begin{array}{ccc}
\lambda+\beta y_{2}+\gamma & -\beta\left(1-x_{2}\right) & 0 \\
\alpha\left(y_{2}+z_{2}-1\right) & \lambda+\alpha x_{2}+\mu+c z_{2} & \alpha x_{2}+c y_{2} \\
0 & 0 & \lambda-\delta+\omega+2 \delta z_{2}
\end{array}\right| \\
& \quad=0 .
\end{aligned}
$$

Solving the characteristic equation, we can get its characteristic roots:

$$
\begin{gathered}
\lambda_{1}=\delta-\omega-2 \delta z_{2}=\omega-\delta \\
\lambda_{2,3}=\frac{-\gamma-\mu-\alpha x_{2}-\beta y_{2}-c z_{2} \pm \sqrt{\Delta}}{2}
\end{gathered}
$$

where

$$
\begin{aligned}
& \Delta=\left(\gamma+\mu+\alpha x_{2}+\beta y_{2}+c z_{2}\right)^{2} \\
& -4\left(\left(\beta y_{2}+\gamma\right)\left(\alpha x_{2}+\mu+c z_{2}\right)\right. \\
& \left.-\alpha \beta\left(1-x_{2}\right)\left(1-y_{2}-z_{2}\right)\right) .
\end{aligned}
$$

From (13) and (21), we have

$$
\lambda_{1}=\delta-\omega-2 \delta z_{2}=\omega-\delta<0 .
$$

$\operatorname{Re} \lambda_{2,3}<0$ is equivalent to

$$
\left(\beta y_{2}+\gamma\right)\left(\alpha x_{2}+\mu+c z_{2}\right)-\alpha \beta\left(1-x_{2}\right)\left(1-y_{2}-z_{2}\right)>0
$$

By the condition $\omega<\delta$ and the above inequality, we have

$$
\begin{aligned}
& \left(\beta y_{2}+\gamma\right)\left(\alpha x_{2}+\mu+c z_{2}\right)-\alpha \beta\left(1-x_{2}\right)\left(1-y_{2}-z_{2}\right) \\
& =\alpha \beta \frac{\omega}{\delta}-\gamma\left(\mu+c-\frac{c \omega}{\delta}\right) \\
& =\gamma\left(\mu+c-\frac{c \omega}{\delta}\right)\left(\mathbf{R}_{0}^{2}-1\right)>0 .
\end{aligned}
$$

Therefore, system (11) is locally asymptotically stable about $\left(x_{2}, y_{2}, z_{2}\right)$. The proof of Theorem 4 is completed.

Remark 5. When $\delta_{1} \neq \delta_{2}$, taking $\mathbf{R}_{0}^{2}=\alpha \beta \omega /\left(c \gamma \delta_{2}+\gamma \delta_{2} \mu-\right.$ $c \gamma \omega)$, for system (11), the conclusions of Theorem 4 are still valid, but the proofs are more complicated. So we will verify them with numerical simulations in the following section.

Remark 6. From the above discussion, we can see that releasing transgenic mosquitoes into wild mosquitoes in the target field can prevent malaria. When $\mathbf{R}_{0}^{2}>1$, we can make the proportions of patients and anopheles steady and malaria will not be massively popular. Most importantly, when $\mathbf{R}_{0}^{2}<1$, there will be only transgenic mosquitoes; patients and anopheles will disappear. That is to say, we can eventually eradicate malaria. It is easy to see when we are unable to change the traditional infection rate and death rate; as long as we increase the amount of transgenic mosquitoes and the birth rate $\delta$ of transgenic mosquitoes bred by wild mosquitoes, then malaria can be eliminated.

\section{Numerical Simulations}

Here mainly for system (11), especially for $\delta_{1} \neq \delta_{2}$, we verify its results with numerical simulations. Assume that the amounts 


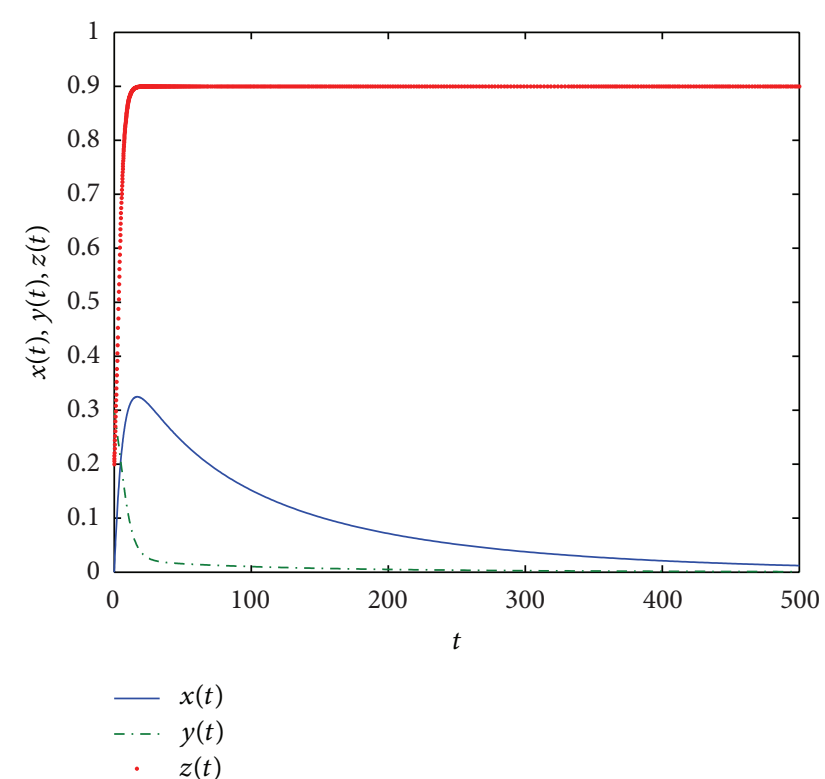

Figure 1: Parameters: $\beta=0.2, \alpha=0.1, \gamma=0.02, \mu=0.05, \omega=$ $0.05, c=0.1$, and $\delta_{1}=\delta_{2}=\delta=0.5$.

of persons and mosquitoes in an isolated target field are constants. According to the discussions in $[8,11,19-21]$ and empirical data, we let the parameters in system (11) take the following ranges as in Table 3.

At the beginning, we assume that there is no patients; the proportion of anopheles and the proportion of transgenic mosquitoes are 0.3 and 0.2 , respectively. That is to say, the initial values of system $(11)$ are $(0,0.3,0.2)$. We take parameter values as $\beta=0.2, \alpha=0.1, \gamma=0.02, \mu=0.05, \omega=0.05, c=$ 0.1 , and $\delta_{1}=\delta_{2}=\delta=0.5$. According to these parameter values, we have

$$
\mathbf{R}_{0}^{2}=\frac{\alpha \beta \omega}{c \gamma \delta+\gamma \delta \mu-c \gamma \omega} \approx 0.714286<1 .
$$

The corresponding result of numerical simulation is shown in Figure 1.

From Theorem 4 and the above parameter values, it is easy to know that system (11) should be stable on $(0,0,0.9)$. From Figure 1, we can see that the simulation result is consistent with the result of Theorem 4. From the curve for anopheles, we can see that releasing the transgenic mosquitoes can effectively reduce anopheles. It follows that releasing the transgenic mosquitoes suppresses the outbreak of malaria in a relatively short time. As to why the patient and anopheles do not disappear immediately after a sharp drop in a short time, that is, it takes a long time that their amounts reach to zero, we think that the male transgenic mosquitoes cannot compete with some excellent wild male mosquitoes and they cannot capture the "heart" of all female anopheles in a short time or mating is a probability event. There is always mating between wild male mosquitoes and female anopheles. But after transgenic mosquitoes released control the rest of anopheles to a very small amount, the anopheles will die

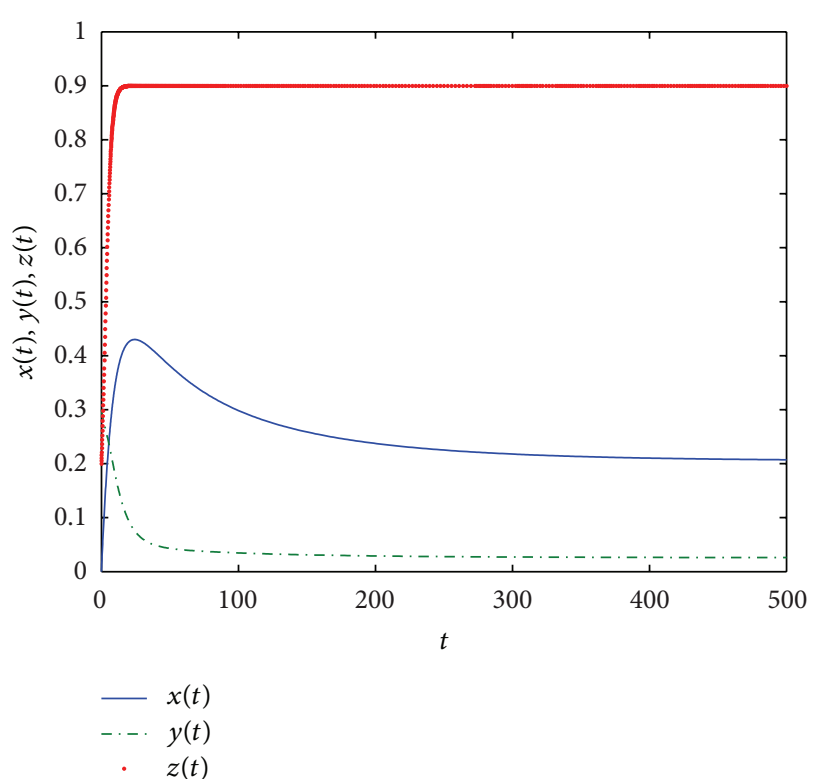

Figure 2: Parameters: $\beta=0.2, \alpha=0.1, \gamma=0.02, \mu=0.05, \omega=$ $0.05, c=0.01$, and $\delta_{1}=\delta_{2}=\delta=0.5$.

themselves one month later due to their limited lifetime. It can be considered that anopheles have been eliminated.

Take $c=0.01$ and keep other parameter values unchanged. That is, we assume that the decrement rate of anopheles due to transgenic male mosquito bred by transgenic mosquito and anopheles is decreased. According to the values, we have

$$
\mathbf{R}_{0}^{2}=\frac{\alpha \beta \omega}{c \gamma \delta+\gamma \delta \mu-c \gamma \omega} \approx 1.69492>1 .
$$

The corresponding result of numerical simulation is shown in Figure 2.

From Theorem 4 and the above parameter values, it is easy to know that system (11) should be stable about $(0.205,0.026,0.9)$ which is an endemic equilibrium. From Figure 2, we can see that the simulation result is also consistent with result of Theorem 4 and parameter $c$ has a distinct impact on the reduction of anopheles. When we decrease $c$, the reduction speed of anopheles becomes significantly slow.

When $\delta_{1} \neq \delta_{2}$, take $\delta_{2}=0.25$ and keep other parameter values in the first numerical simulation unchanged. We have

$$
\mathbf{R}_{\mathbf{0}}^{2}=\frac{\alpha \beta \omega}{c \gamma \delta_{2}+\gamma \delta_{2} \mu-c \gamma \omega} \approx 1.53846>1 .
$$

The corresponding result of numerical simulation is shown in Figure 3.

Take $\delta_{1}=0.1$ and $\delta_{2}=0.5$ and keep other values unchanged; we have

$$
\mathbf{R}_{\mathbf{0}}^{2}=\frac{\alpha \beta \omega}{c \gamma \delta_{2}+\gamma \delta_{2} \mu-c \gamma \omega} \approx 0.714286<1 .
$$

The corresponding result of numerical simulation is shown in Figure 4. 
TABLE 3: Ranges of the parameters in system (11).

\begin{tabular}{llc}
\hline Parameter & Description & Range (per day) \\
\hline$\beta$ & Incidence rate of malaria due to biting & $0.1-0.5$ \\
$\alpha$ & Efficiency of infection in mosquitoes by biting patients & $0.01-0.2$ \\
$\mu$ & Death rate of anopheles & $0.05-0.5$ \\
$\gamma$ & Recovery rate of patients & $0.01-0.1$ \\
$c$ & Decrement rate of anopheles due to transgenic mosquitoes bred by transgenic & $0.01-0.2$ \\
$\omega$ & mosquitoes and anopheles & $0.05-0.5$ \\
$\delta_{1}$ & Death rate of transgenic mosquitoes & $0.1-0.5$ \\
$\delta_{2}$ & Birth rate of transgenic mosquitoes bred by transgenic mosquitoes and wild & $0.1-0.5$ \\
\hline
\end{tabular}

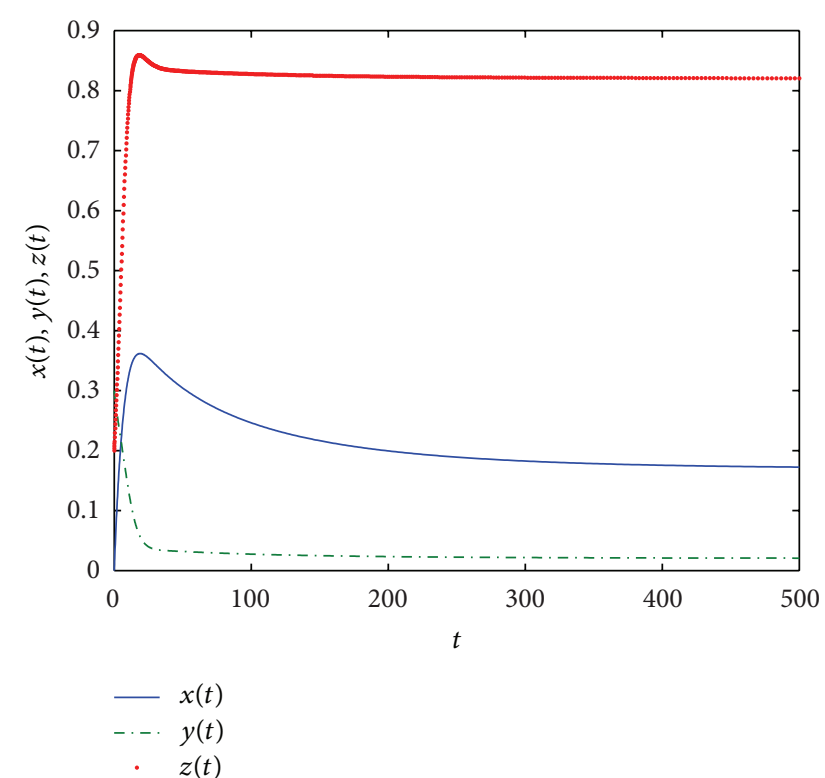

Figure 3: Parameters: $\beta=0.2, \alpha=0.1, \gamma=0.02, \mu=0.05, \omega=$ $0.05, c=0.1, \delta_{1}=0.5$, and $\delta_{2}=0.25$.

From Figure 3, we can see that $x(t) \rightarrow x_{2}, y(t) \rightarrow$ $y_{2}$, and $z(t) \rightarrow z_{2}$ as $t \rightarrow \infty$. From Figure 4 we can see that $x(t) \rightarrow 0, y(t) \rightarrow 0, z(t) \rightarrow z_{1}$ as $t \rightarrow \infty$. That is to say, when $\delta_{1} \neq \delta_{2}$, the results of Theorem 4 remain valid.

Take $\alpha=0.14, \delta_{1}=0.1$, and $\delta_{2}=0.5$ and keep other values unchanged; we have

$$
\mathbf{R}_{\mathbf{0}}^{2}=\frac{\alpha \beta \omega}{c \gamma \delta_{2}+\gamma \delta_{2} \mu-c \gamma \omega}=1 .
$$

The corresponding result of numerical simulation is shown in Figure 5.

Set $\alpha=0.14$ and $\delta_{1}=\delta_{2}=\delta=0.5$ and keep other values unchanged; we have

$$
\mathbf{R}_{\mathbf{0}}^{2}=\frac{\alpha \beta \omega}{c \gamma \delta+\gamma \delta \mu-c \gamma \omega}=1 .
$$

The corresponding result of numerical simulation is shown in Figure 6.

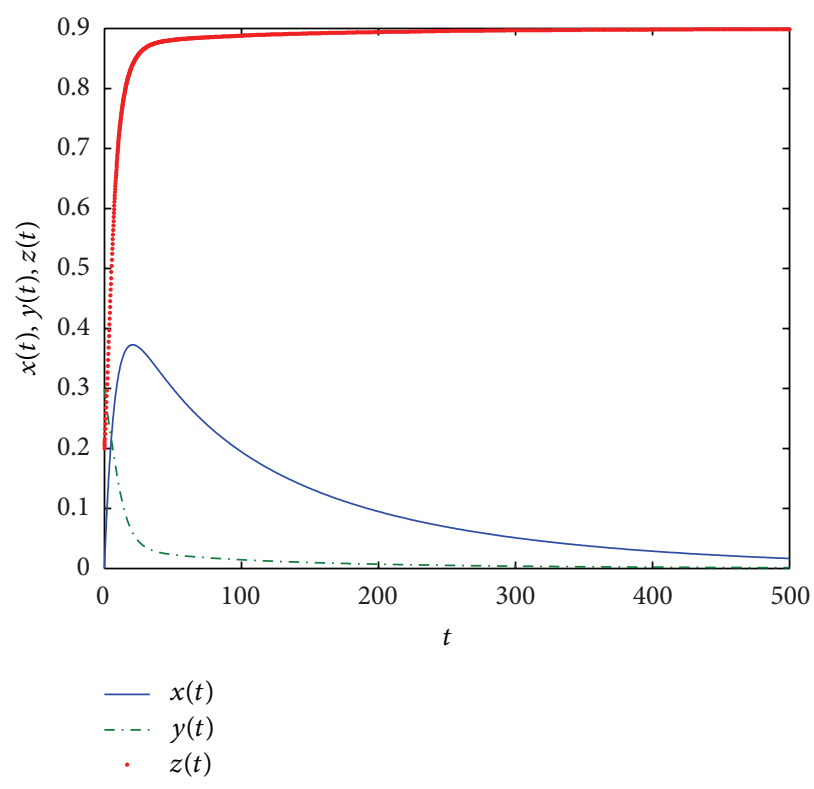

FIgURE 4: Parameters: $\beta=0.2, \alpha=0.1, \gamma=0.02, \mu=0.05, \omega=$ $0.05, c=0.1, \delta_{1}=0.1$, and $\delta_{2}=0.5$.

For the above two numerical simulations, we increase the value of $\alpha$; that is to say, we assume that the efficiency of patient infecting mosquito by biting is greater. From Figures 5 and 6 , we can see that anopheles will become more and the decreasing speed of patients will become relatively slow. But malaria and anopheles will still disappear eventually. That is to say, for $\delta_{1} \neq \delta_{2}$ and $\delta_{1}=\delta_{2}$, when $\mathbf{R}_{\mathbf{0}}^{2}=1, x(t) \rightarrow$ $0, y(t) \rightarrow 0$, and $z(t) \rightarrow z_{1}$ as $t \rightarrow \infty$.

\section{Conclusion and Prospect}

In this paper we firstly establish system (1) with transgenic mosquitoes released at a fixed proportion and then establish system (11) with transgenic mosquitoes released at a changeable proportion. For these models, we obtain a diseasefree equilibrium and an endemic equilibrium. We prove theoretically and verify our conclusions of Theorems 1 and 4 with numerical simulations. For $\delta_{1} \neq \delta_{2}$ and $\mathbf{R}_{\mathbf{0}}^{2}=1$, we 


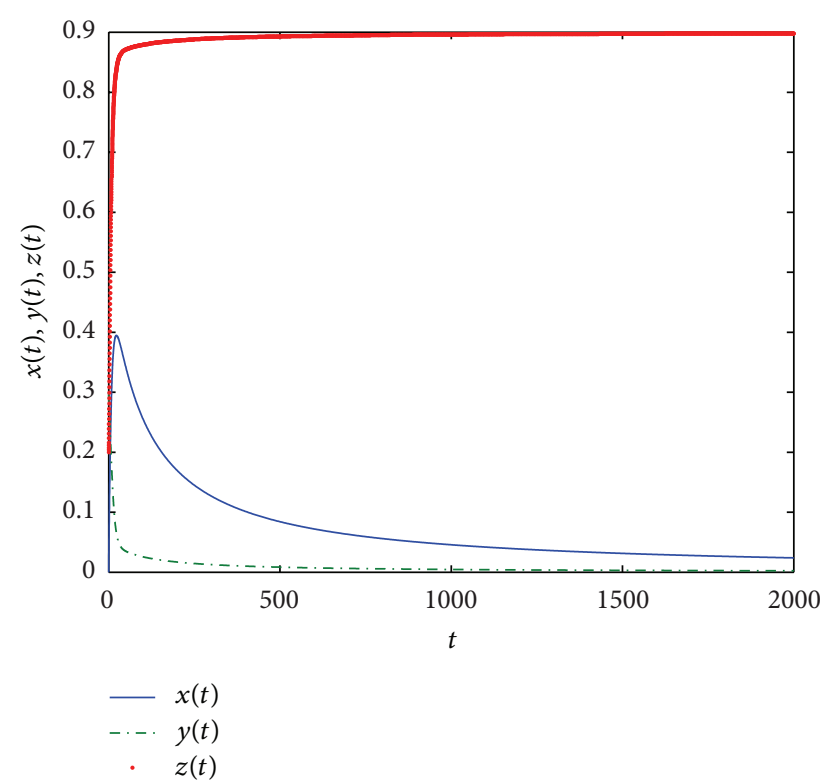

FIgURE 5: Parameters: $\beta=0.2, \alpha=0.14, \gamma=0.02, \mu=0.05, \omega=$ $0.05, c=0.1, \delta_{1}=0.1$, and $\delta_{2}=0.5$.

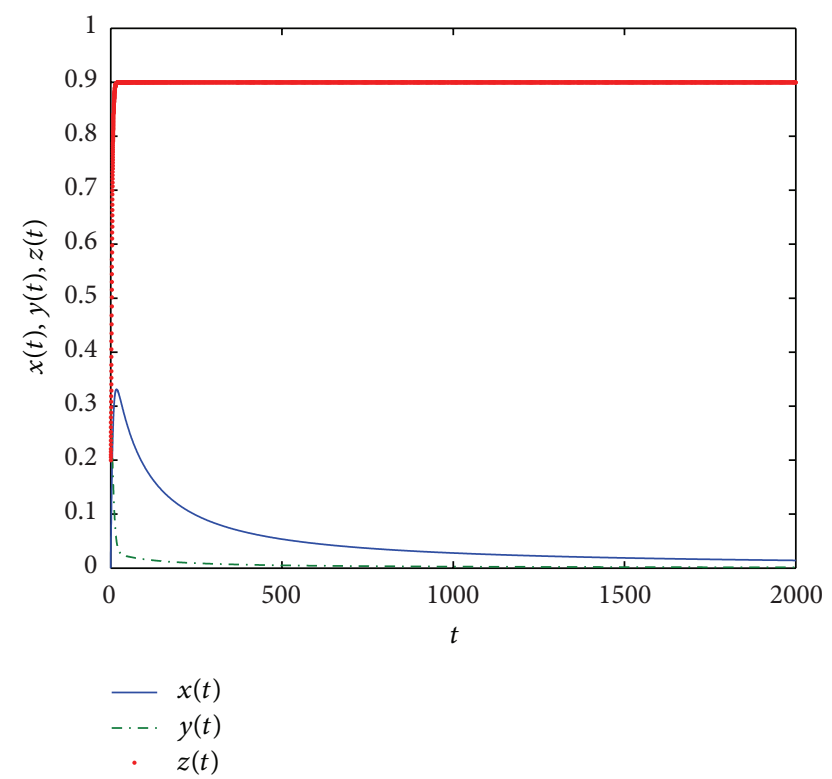

Figure 6: Parameters: $\beta=0.2, \alpha=0.14, \gamma=0.02, \mu=0.05, \omega=$ $0.05, c=0.1, \delta_{1}=0.1$, and $\delta_{2}=0.5$.

do not theoretically prove the conclusions of Theorem 4, but we verify them with simulations. From Figures 3, 4, 5, and 6, we can see our results are also valid. We also have a unified conclusion for system (1) and system (11). That is, if $\mathbf{R}_{\mathbf{0}}^{\mathbf{1}}<1$ or $\mathbf{R}_{0}^{2}<1$, it implies that malaria will be eliminated; if $\mathbf{R}_{0}^{1}>1$ or $\mathbf{R}_{0}^{2}>1$, malaria will become an epidemic disease in the target field.

The models in this paper are simpler and more ideal. For example, we do not take the incubation period of malaria into account and only consider the one-time delivery of transgenic mosquitoes. The factors involved in our models are incomplete and we only considered the local asymptotic stabilities of systems. In order to make up for these deficiencies and establish more realistic models, we think that we can choose different birth function according to the actual situation and take more factors into account, such as susceptible, wild mosquito population, recovered patients, latency period, and environmental factor.

Although our dynamic models have many disadvantages, they are continuous differential equations compared with the existing models which were discrete-differential equations that only considered the competition between two classes of mosquito populations. In addition, our epidemic models for transgenic mosquitoes released at changeable proportion are considered by few researchers. Two models in this paper are consistent with the actual situation and they are relatively complete, various, and comprehensive for the research and applications of transgenic mosquito in malaria transmission.

\section{Conflict of Interests}

The authors declare that there is no conflict of interests regarding the publication of this paper.

\section{Acknowledgments}

This work is supported by Hunan Provincial Natural Science Foundation of China (12jj4012) and Research Project of National University of Defense Technology (JC12-02-01).

\section{References}

[1] F. A. Collins and A. A. James, "Genetic modifications of mosquitoes," Science Medicine, vol. 3, pp. 52-61, 1996.

[2] G. K. Christophides, "Transgenic mosquitoes and malaria transmission," Cellular Microbiology, vol. 7, no. 3, pp. 325-333, 2005.

[3] F. Gould, K. Magori, and Y. X. Huang, "Genetic strategies for controlling mosquito-borne diseases," American Scientist, vol. 94, no. 3, pp. 238-246, 2006.

[4] S. Levy, "Mosquito modifications: new approaches to controlling malaria," BioScience, vol. 57, no. 10, pp. 816-821, 2007.

[5] J. V. Lavery, L. C. Harrington, and T. W. Scott, "Ethical, social, and cultural considerations for site selection for research with genetically modified mosquitoes," American Journal of Tropical Medicine and Hygiene, vol. 79, no. 3, pp. 312-318, 2008.

[6] B. G. J. Knols, H. C. Bossin, W. R. Mukabana, and A. S. Robinson, "Transgenic mosquitoes and the fight against malaria: managing technology push in a turbulent GMO world," The American Journal of Tropical Medicine and Hygiene, vol. 77, no. 6, pp. 232-242, 2007.

[7] L. M. Dai, The influence of resistant mosquitoes to malaria dynamical system [M.S. thesis], Chongqing, China, 2009, Southwest University.

[8] R. Antia, A. Yates, and J. C. de Roode, "The dynamics of acute malaria infections. I. Effect of the parasite's red blood cell preference," Proceedings of the Royal Society B, vol. 275, no. 1641, pp. 1449-1458, 2008. 
[9] N. R. Chitnis, Using mathematical models in controlling the spread of malaria [Ph.D. thesis], the university of Arizona, Tucson, Arizona, 2005.

[10] J. Li, "Simple stage-structured models for wild and transgenic mosquito populations," Journal of Difference Equations and Applications, vol. 15, no. 4, pp. 327-347, 2009.

[11] J. Li, "Simple mathematical models for interacting wild and transgenic mosquito populations," Mathematical Biosciences, vol. 189, no. 1, pp. 39-59, 2004.

[12] J. Li, "Discrete time models with mosquitoes carrying genetically modified bacteria," Mathematical Biosciences, vol. 240, no. 1, pp. 35-44, 2012.

[13] J. Li, S. B. Ai, and J. L. Lu, "Mosquito-stage-structuried Malaria models and their global dynamics," SIAM Journal of Applied Mathematics, vol. 27, pp. 1223-1237, 2012.

[14] J. Li, "Modelling of transgenic mosquitoes and impact on malaria transmission," Journal of Biological Dynamics, vol. 5, no. 5, pp. 474-494, 2011.

[15] W. O. Kermack and A. G. Mckendrick, "A contribution to the mathematical theory of epidemics," Proceeding of the Royal Society of London A, vol. 115, no. 772, pp. 700-721, 1927.

[16] V. Lakshmikantham, Nonlinear Systems and Applications, Princeton University Press, Princeton, NJ, USA, 1976.

[17] L. S. Chen, X. Z. Meng, and J. J. Jiao, Biodynamics, Science Press, Beijing, China, 2009.

[18] B. H. Kim, H. K. Kim, and S. J. Lee, "Experimental analysis of the blood-sucking mechanism of female mosquitoes," The Journal of Experimental Biology, vol. 214, no. 7, pp. 1163-1169, 2011.

[19] F. Oduro, G. A. Okyere, and G. T. Azu-Tungmah, "Transmission dynamics of Malaria in ghana," Journal of Mathematics Research, vol. 4, no. 6, pp. 22-33, 2012.

[20] S. Ruan, D. Xiao, and J. C. Beier, "On the delayed RossMacdonald model for malaria transmission," Bulletin of Mathematical Biology, vol. 70, no. 4, pp. 1098-1114, 2008.

[21] A. F. Harris, D. Nimmo, A. R. McKemey et al., "Field performance of engineered male mosquitoes," Nature Biotechnology, vol. 29, no. 11, pp. 1034-1037, 2011. 


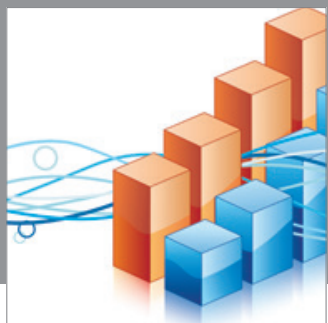

Advances in

Operations Research

mansans

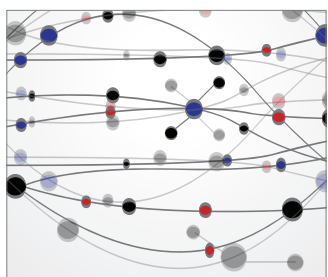

The Scientific World Journal
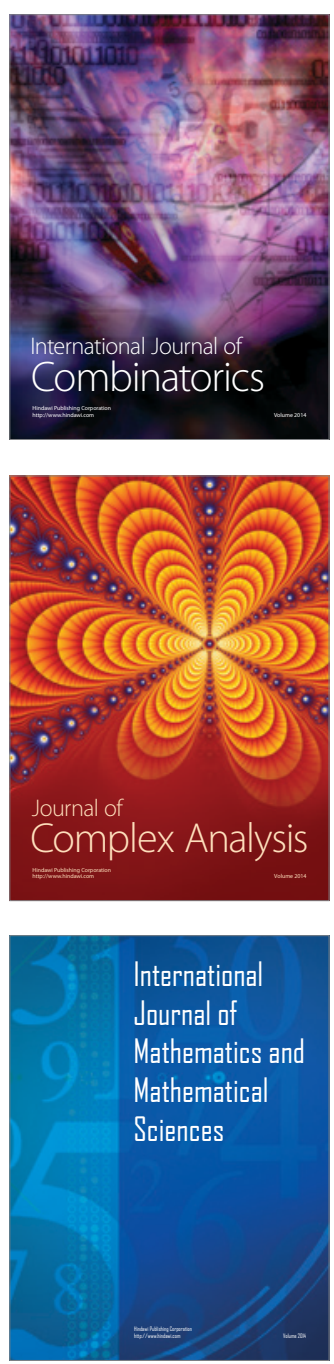
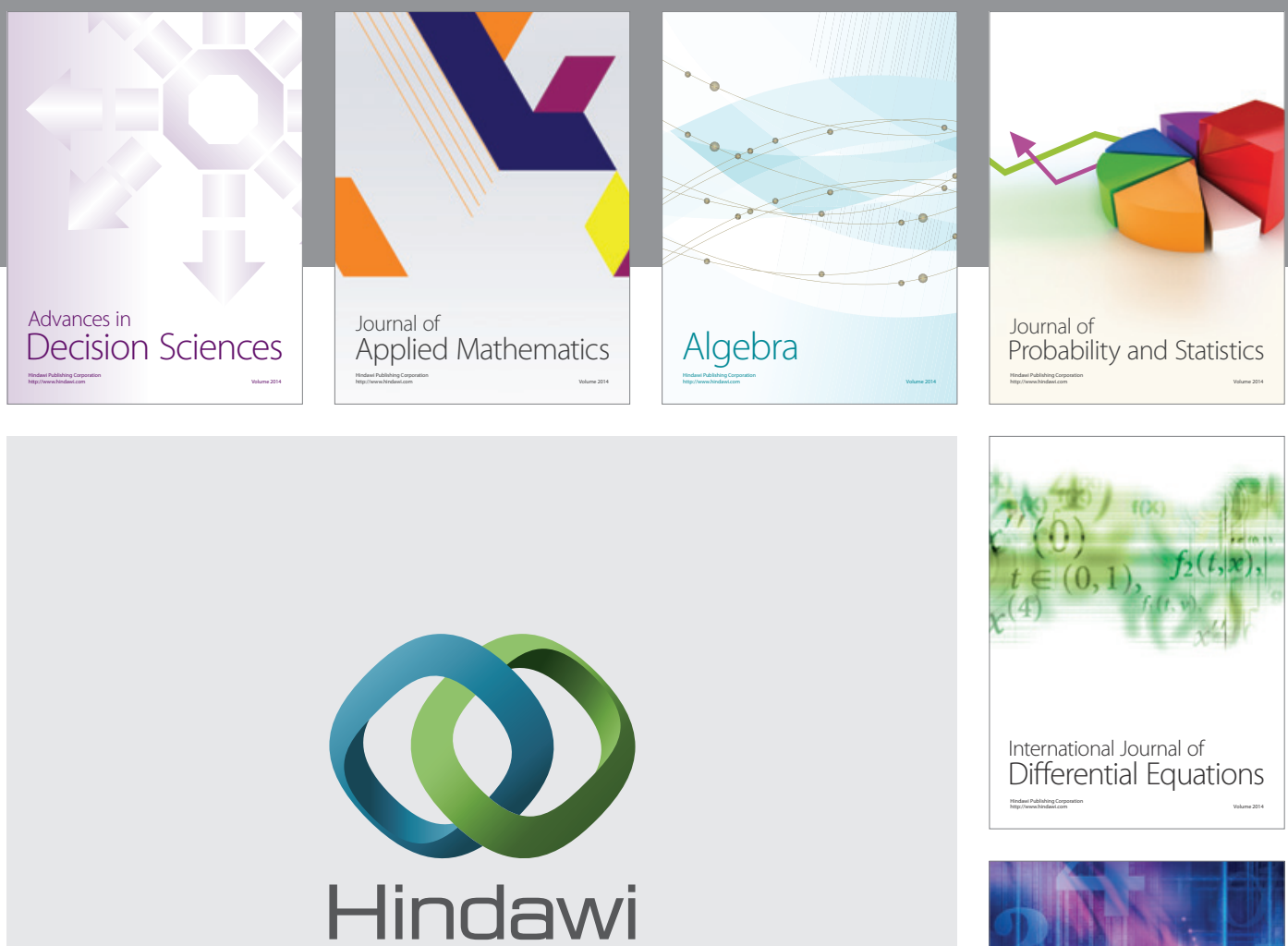

Submit your manuscripts at http://www.hindawi.com
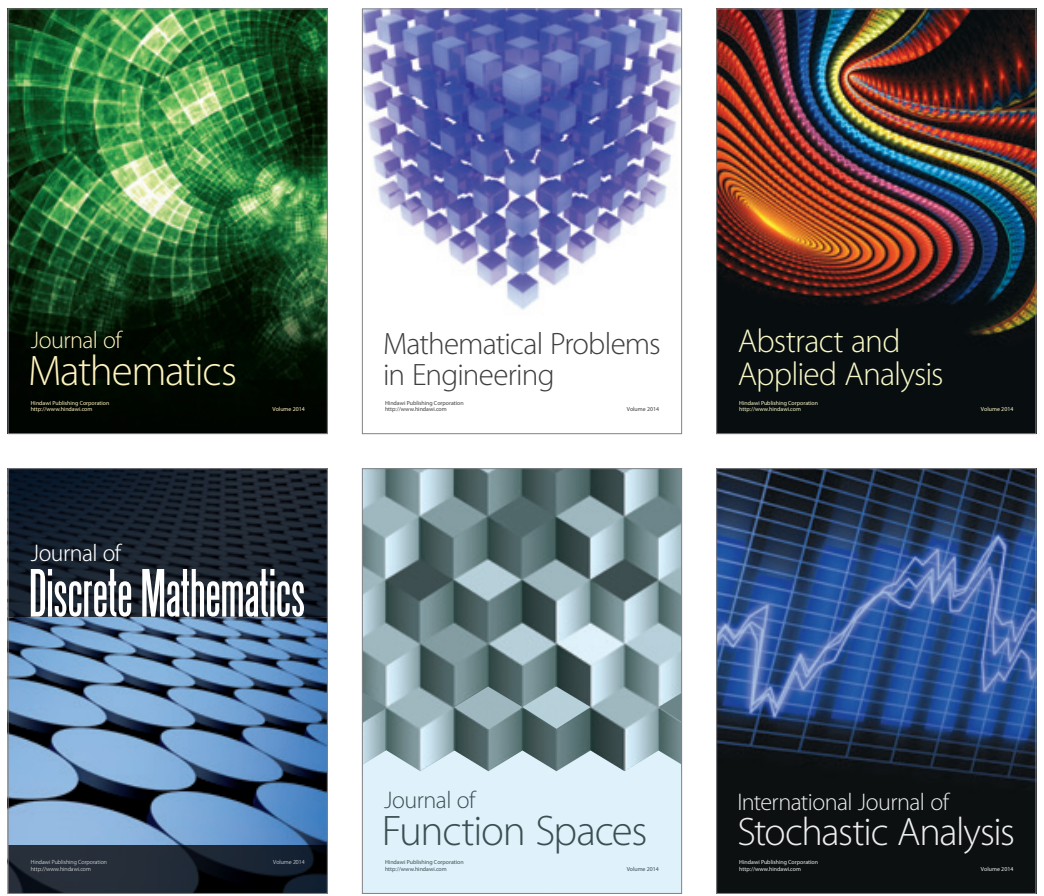

Journal of

Function Spaces

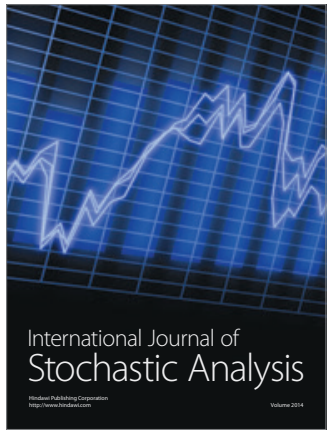

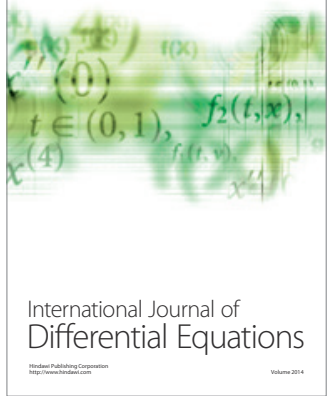
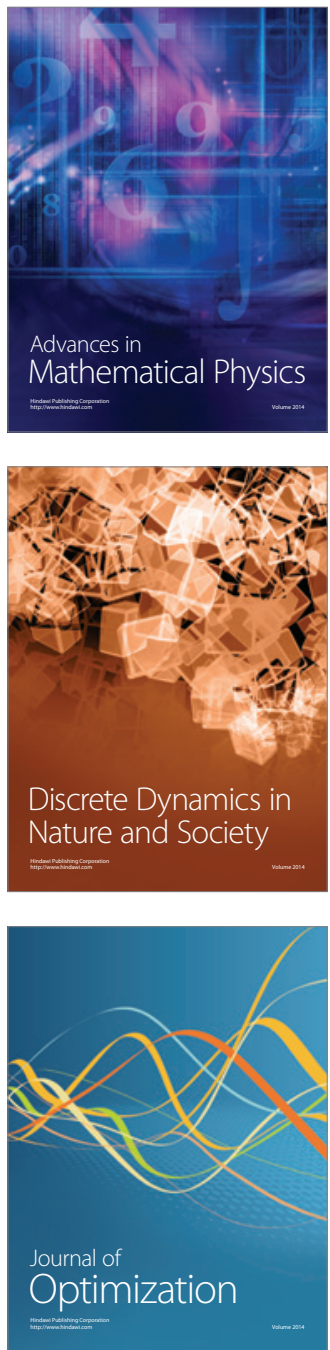